\title{
Generation of an inducible system to express polo-like kinase, Cdc5 as TAP fusion protein during meiosis in Saccharomyces cerevisiae
}

\author{
Rajni Vaid $^{1} \cdot$ Kamal Dev $^{1} \cdot$ Michael Lichten ${ }^{2} \cdot$ Anuradha Sourirajan $^{1}$
}

Received: 9 June 2016/Accepted: 22 August 2016/Published online: 30 August 2016

(c) The Author(s) 2016. This article is published with open access at Springerlink.com

\begin{abstract}
Tandem affinity purification (TAP) is a highly efficient method for isolation of protein complexes from endogenous biological macromolecules. TAP system consists of dual affinity tags that facilitates the sequential purification of the desired proteins expressed at their low levels in vivo. Polo-like kinases (PLK) are serine/threonine protein kinases that are the crucial regulators of cell cycle. Cdc5, the solitary PLK in budding yeast Saccharomyces cerevisiae, has diverse array of targets in cell cycle. The present study was undertaken to construct an estrogen-inducible system for expression of Cdc5-TAP to isolate the substrates of Cdc5 during meiosis, particularly, pachytene stage of meiosis I. Two yeast strains were constructed CDC5-IN (ndt804 pGAL1-CDC5-TAP) and Cdc5-kinase inactive mutant (ndt804 $p$ GAL1-cdc5-N209A-TAP). The estrogen-inducible expression of Cdc5-TAP and cdc5N209A-TAP was validated by Western analysis. The systems would serve as a valuable tool for purification of substrates binding to Cdc5-TAP by TAP affinity chromatography.
\end{abstract}

Electronic supplementary material The online version of this article (doi:10.1007/s13205-016-0503-x) contains supplementary material, which is available to authorized users.

Anuradha Sourirajan

asourirajan@gmail.com

Michael Lichten

mlichten@helix.nih.gov

1 Faculty of Applied Sciences and Biotechnology, Shoolini University, Solan, Himachal Pradesh 173212, India

2 Laboratory of Biochemistry and Molecular Biology, Center for Cancer Research, National Cancer Institute, Bethesda, MD 20892, USA
Keywords Tandem affinity purification - Fusion protein · Saccharomyces cerevisiae - Meiosis - Polo-like kinase . Cdc5 - Kinase dead - Estrogen inducible - Pachytene . Meiotic recombination $\cdot$ Joint molecules $(\mathrm{JM}) \cdot$ Substrates

\section{Introduction}

Polo-like kinases are serine threonine kinases characterized by the presence of protein kinase domain at the $\mathrm{N}$-terminus, and a polo box domain (PBD) at the C-terminus, the latter playing a role in the modulation of kinase activity and substrate recognition (Lee et al. 2005). PLKs spatiotemporally regulate and coordinate mitosis and meiosis. A single PLK exists in Saccharomyces cerevisiae (Cdc5), while multiple PLKs have been identified in other organisms, except plants (Vaid et al. 2016). In S. cerevisiae, Cdc5, the solitary PLK regulates G2/M phase transition, metaphase to anaphase transition during mitosis, exit from mitosis and cytokinesis (Nigg 1998; Lee et al. 2005; Vaid et al. 2016). In eukaryotic systems, meiosis is imperative for genetic diversity and meiotic recombination. During meiosis, bi-phasic division takes place, wherein during the reductional division (meiosis I) homologous chromosomes segregate followed by equational division (meiosis II) producing four haploid gametes (Hochwagen and Amon 2006; Simchen 2009; Börner and Cha 2015). Cdc5 is a central regulator of meiosis I in S. cerevisiae (Attner et al. 2013; Vaid et al. 2016). Cdc5 regulates the meiotic functions in prophase I, including exit from pachytene, mono-orientation of the sister kinetochores, removal of cohesins from chromosomal arms at metaphase I and anaphase I, respectively, followed by meiotic divisions (Sharon and Simchen 1990; Clyne et al. 2003; Lee and Amon 2003a, b; Vaid et al. 2016). In S. cerevisiae, a meiosis-specific transcription factor, $\mathrm{Ndt} 80$, is essential for meiotic progression (Chu and Herskowitz 1998). 
Cells lacking Ndt80 arrest at pachytene and fail to complete meiotic divisions (Benjamin et al. 2003). During meiosis, transcription of $C D C 5$ is activated by Ndt80. Similar to Ndt80, Cdc5 has been shown to be necessary and sufficient for pachytene exit in meiosis I, raising the possibility of some targets of $\mathrm{Cdc} 5$ to be required for pachytene exit (Clyne et al. 2003; Lee and Amon 2003a, b; Sourirajan and Lichten 2008). However, the downstream targets of $\mathrm{Cdc} 5$ in pachytene exit have not been deciphered. Some of the targets of Cdc5 have been already identified, like Rec8, Mam1, and Ime2, none of which has a role in pachytene exit (Clyne et al. 2003; Lee et al. 2008; Sourirajan and Lichten 2008; Attner et al. 2013). There is still an array of unexplored targets, whose discovery could provide an insight into the role of Cdc5 in pachytene exit in specific, and meiosis as a whole.

The present study was initiated to develop an amenable tool for identification of the substrates of the PLK, Cdc5 during yeast meiosis. An estrogen-inducible system for Cdc5 expression during specific phase in meiosis was generated as described in previous studies (Benjamin et al. 2003; Sourirajan and Lichten 2008). The inducible system was combined with the TAP-tagging of Cdc5 to identify the targets of Cdc5 during yeast meiosis (Puig et al. 2001). The system was functionally validated by Western analysis.

Tandem affinity purification (TAP) tag is an affinity chromatography-based rapid and generic system of protein purification, which is further analyzed with mass spectrometry to identify the protein ensembles that are part of any functional proteome (Rigaut et al. 1999; Puig et al. 2001; Xu et al. 2010). The scope of TAP method in yeast allows the purification of TAP-tagged protein and its associated components using dual affinity system (Rigaut et al. 1999). The C-terminal TAP insertion cassette consists of two domains, proximally a calmodulin-binding peptide, a tobacco etch virus (TEV) protease cleavage site (Dougherty et al. 1989; Kapust et al. 2001) in the center and an IgG binding domain of Staphylococcus aureus protein A at the distal end (Uhlen et al. 1983; Puig et al. 2001). The desired protein with C-terminal TAP tag is sequentially purified under mild conditions on IgG matrix followed by the elution with TEV protease, and then passed through the calmodulin resin in the presence of $\mathrm{Ca}^{2+}$ ions and finally eluting the protein of interest in presence of ethylene glycol tetra acetic acid (EGTA) (Puig et al. 2001; Van Driessche et al. 2005).

\section{Methods}

Chemicals and reagents of biochemical and molecular biology grade were procured from Himedia Labs, India; MP Biomedicals, USA; Fermentas Inc. USA; Bio-Rad,
USA. Antibodies used in this study were purchased from Thermo scientific, USA and Santa Cruz Biotechnologies, Inc. USA.

\section{Strains and plasmids}

All the $S$. cerevisiae strains used in this study were of SK1 genotype (Kane and Roth 1974; Allers and Lichten 2001). The genomic-tagged yeast strains containing CDC5-TAP (with C-terminus TAP tag) in the SK1 background were a kind gift of Dr. Wolfgang Zachariae, Max Planck Institute of Molecular Cell Biology and Genetics, Germany (Matos et al. 2008). The CDC5-TAP yeast strains containing the $U R A 3-A R G 4$ recombinant inserts were constructed using genetic crosses. The genotypes of the yeast strains used in this study are given the Table S1 (Supplementary material).

Two integrant plasmids pMJ830 and pMJ840 were used to generate $C D C 5-I N$ and $c d c 5-N 209 A-I N$ yeast strains (Supplementary material). S. cerevisiae strains were grown on YEPD medium (1\% yeast extract, $2 \%$ peptone, and $2 \%$ dextrose), supplemented with $0.8 \%$ adenine sulfate at $30{ }^{\circ} \mathrm{C}$. Nutrient broth (NB, Himedia Labs, Mumbai) with $100 \mu \mathrm{g} / \mathrm{ml}$ ampicillin was used to culture and grow the bacterial strains of $E$. coli DH5 $\alpha$ harboring pMJ830 and pMJ840 at $37{ }^{\circ} \mathrm{C}$. Plasmid DNA of pMJ830 and pMJ840 were isolated and digested with SnaB I at $37{ }^{\circ} \mathrm{C}$ for $1 \mathrm{~h}$ and used in transformations. Transformants were grown and selected on hygromycin selection medium (YEPD medium containing $300 \mu \mathrm{g} / \mathrm{ml}$ of hygromycin) at $30{ }^{\circ} \mathrm{C}$.

\section{Construction of CDC5-IN (ndt80A pGAL1-CDC5- TAP) and $c d c 5-N 209 A-I N$ (ndt80 4 pGAL1- $c d c 5 N 209 A-T A P)$ diploid strains}

\section{Transformation of the yeast strain with SnaB I digested pMJ830 and pMJ840}

Construction of CDC5-IN and $c d c 5-N 209 A-I N$ diploid strains was done through genomic integration approach. $5 \mathrm{ml}$ of YEPD broth was inoculated with single colony of the haploid parental yeast strain S3561 (Table S1) and cultured at $30{ }^{\circ} \mathrm{C}$ for $16 \mathrm{~h}$. $0.5 \mathrm{ml}$ of the culture $\left(A_{600} \sim 2.0\right)$ of $\mathrm{S} 3561$ was harvested and the yeast cells were transformed with two microgram of each of the SnaB I digested and linearized plasmids of pMJ830 and pMJ840 using EZ-transformation method (MP Biomedicals, USA). The transformation mixtures were plated on hygromycin selection media (YEPD containing $300 \mu \mathrm{g} / \mathrm{ml}$ hygromycin) and incubated at $30{ }^{\circ} \mathrm{C}$ for $2-3$ days until transformant colonies were observed. 


\section{Verification of the transformants}

The integration of the plasmid at the $C D C 5$ locus was verified by PCR reactions using gene specific and vector specific primers (Table S2). The DNA of the haploid transformants was isolated (Sambrook et al. 2009) and the PCR was set with three different primer sets as $111 \mathrm{f}-$ as $113 r$, as $65 f-a s 113 r$, and as65f-as $106 r$, respectively. The sequence of each primer is given in Table S2 (Supplementary material). The reaction mixture of PCR contained Dream Taq (2U), reaction buffer 1X, dNTP mix $(100 \mu \mathrm{M}$ each), primers $\left(1 \mu \mathrm{M}\right.$ each). PCR was run at $94{ }^{\circ} \mathrm{C}, 2 \mathrm{~min}$ (initial denaturation); 35 cycles each of denaturation, annealing, and extension; at $94{ }^{\circ} \mathrm{C}, 2 \mathrm{~min} ; 58{ }^{\circ} \mathrm{C}, 30 \mathrm{~s}$, and $72{ }^{\circ} \mathrm{C}, 2 \min 30 \mathrm{~s}$, respectively, with a final extension of $72{ }^{\circ} \mathrm{C}, 10 \mathrm{~min}$.

\section{Generation of ASd1 (CDC5-IN) and ASd2 (cdc5- N209-IN) diploids}

The resulting CDC5-TAP-IN (ASh1) and $c d c 5-N 209 A-T A P$ (ASh2) haploid transformant strains after confirming by PCR were crossed with the other wild-type parental strain of opposite mating type (Mat $\alpha$; S3565) on YEPD plates to result in CDC5-TAP-IN (ASd1) and cdc5-N209A-TAP-IN (ASd2) diploid strains, respectively. The genotype of the strains is described in Table S1 (Supplementary material).

\section{Inducible expression of $C d c 5-T A P$ in meiosis}

\section{Induction of meiosis (Sporulation)}

The $C D C 5-I N$ and $c d c 5-N 209 A-I N$ diploid strains were first grown in YEPD medium supplemented with $0.8 \%$ adenine sulfate at $30{ }^{\circ} \mathrm{C}$ for $24 \mathrm{~h}$. For induction of meiosis, yeast cells were subjected to nitrogen deprivation by transferring to $1 \%$ potassium acetate at $30{ }^{\circ} \mathrm{C}$ with vigorous shaking at $350 \mathrm{rpm}$, as described (Goyon and Lichten 1993; Allers and Lichten 2001). The culture was incubated for $7 \mathrm{~h}$ at $30{ }^{\circ} \mathrm{C}$ (by when most of the cells will complete pachytene and arrest at pachytene stage, owing to the absence of Ndt80) (Allers and Lichten 2001; Sourirajan and Lichten 2008). After $7 \mathrm{~h}$, Cdc5-TAP expression was induced in a portion of the meiotic culture by the addition of $1 \mu \mathrm{M} \beta$ estradiol (ED). 5-10 $\mathrm{ml}$ of meiotic yeast cells were collected from uninduced samples and after $2 \mathrm{~h}$ past induction for western analysis.

\section{Preparation of protein extracts and Western blotting}

Proteins were extracted using $20 \%$ tri-chloroacetic acid (TCA) and estimated by Bradford method (Bradford 1976).
The protein extracts ( $15 \mu \mathrm{g}$ of total protein) were resolved on $10 \%$ SDS-polyacrylamide gel and electroblotted onto the nitrocellulose membrane. The blot was incubated in blocking solution containing $5 \%$ non-fat dried milk for $1 \mathrm{~h}$. Membrane was then incubated with 1:1000 dilution of rabbit anti-TAP tag antibodies (Thermo Scientific, USA) overnight at $4{ }^{\circ} \mathrm{C}$. Membrane was then incubated with 1:7500 dilution of anti-rabbit $\operatorname{IgG}$ conjugated to horseradish peroxidase (Santa Cruz Biotechnologies, USA) for $1 \mathrm{~h}$ and developed using enhanced chemiluminescence kit (ECL, Bio-Rad, Inc. USA).

\section{Results and discussion}

\section{Generation of an estradiol-inducible CDC5-TAP expression system to purify the substrates of $\mathrm{Cdc5}$ during meiosis}

The experimental system consists of the inducible and Ndt80-independent expression of CDC5-TAP in $n d t 80 \Delta$ cells during meiosis (Sourirajan and Lichten 2008). Yeast mutants lacking NDT80 (ndt80 $\Delta)$ arrest at pachytene (Xu et al. 1995), thereby serving as a synchronous population of meiotic cells in pachytene stage. The $C D C 5$ gene consists of tandem affinity purification (TAP) tag at the end of the gene, which will produce a Cdc5-TAP protein with a TAP tag at the C-terminus. The TAP tag will facilitate the purification of Cdc5-bound substrates in meiosis. Expression of CDC5-TAP from $p G A L 1$ is achieved using a chimeric Gal4:estrogen receptor (ER) transcription factor (Gal4:ER), which in turn, is activated by the addition of the ligand inducer, estradiol (ED) (Fig. 1).

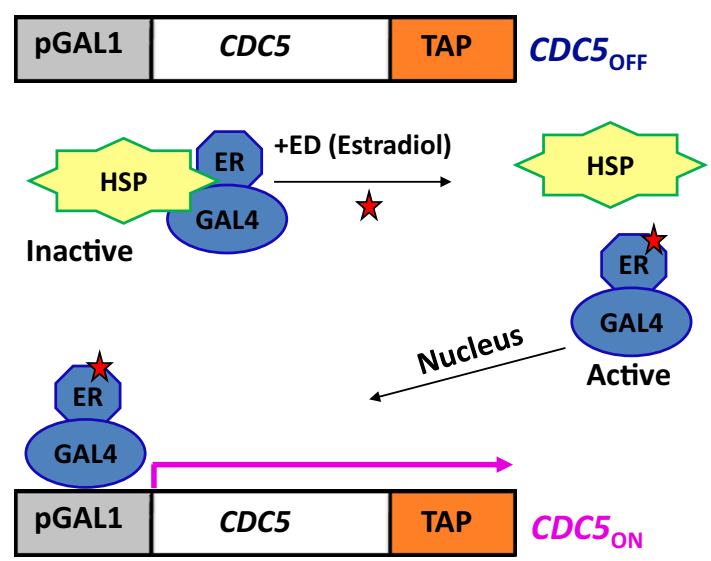

Fig. 1 Estrogen-inducible system to achieve Ndt80-independent expression of $C D C 5-T A P$ during meiosis. In the absence of the ligand, estradiol (ED), CDC5-TAP gene is off. Addition of ED activates Gal4:ER, which translocates to nucleus and turns on $C D C 5$ expression from $p G A L 1$ promoter

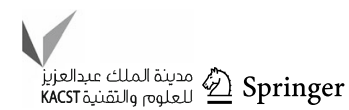




\section{Construction of yeast strains for inducible expression of Cdc5-TAP and cdc5-N209A-TAP during meiosis}

To construct the CDC5-IN (ndt80 $p$ GAL1-CDC5-TAP) diploid strain, genomic integration approach was adopted. In the current strategy, one of the haploid parental strains (S3561) containing CDC5-TAP was transformed with SnaB I linearised integrant plasmid, containing $p G A L 1$ CDC5 (pMJ830). The plasmid also contains hphMX4 marker cassette, which encodes for resistance to hygromycin and thus, the transformants could be selected by plating on hygromycin media (Fig. 2a). The homologous recombination of the integrant plasmid with the genomic CDC5-TAP resulted in the generation of haploid parental strain ASh1, with tandem copies of $C D C 5$ gene, one under its own promoter and the CDC5-TAP under $p G A L 1$ promoter (Fig. 2a).

An additional strain $c d c 5-N 209 A-T A P-I N$ (kinase-dead inducible strain) was constructed using the same strategy as described above, except for the transformation with linearised integrant plasmid with SnaB I enzyme, containing $p G A L 1-c d c 5-N 209 A$ (pMJ840) (Fig. 2). The rationale for the construction of the kinase-dead version of the CDC5-IN strain was based on the fact that the interaction of catalytically inactive Cdc5 kinase (cdc5-N209A) with the substrate/s will be long-lived compared to its wild-type counterpart kinase enzyme, thereby enhancing the efficiency of co-purification of substrates of Cdc5 kinase during TAP-purification. After 3 days of incubation, isolated colonies of transformants were observed $(\sim 20$ per plate). On the other hand, no growth was observed in plates with untransformed parental host strain (S3561) (Fig. 2b). About 8-10 colonies of the transformants were picked and further purified by two rounds of subculturing on the hygromycin selection media. The transformants were selected and used for subsequent experiments.

\section{PCR confirmation of transformants}

The transformants Ash1 and Ash2 were confirmed by PCR with three different sets of primers to verify the plasmid integration of $p$ GAL1-CDC5-TAP (pMJ830) and $p G A L 1$ $c d c 5-N 209-T A P$ (pMJ840) as described in the "Methods". As expected, the primer set as $111 \mathrm{f}-$ as $113 \mathrm{r}$ produced a PCR product of $\sim 2.1 \mathrm{~kb}$ for the wild type, ASh1 and ASh2 (transformants) (Fig. 3a). The primer sets as65f-as113r and as65f-as106r amplified products of $\sim 3.3 \mathrm{~kb}$ and $750 \mathrm{bp}$, respectively, in the transformants, whereas no amplification was observed in the untransformed yeast strain, S3561, with either of the primer sets (Fig. 3b, c). Both ASh1 and ASh2 strains were crossed with the other parental strain of opposite mating type (S3565), which resulted in the diploid strains ASd1 (CDC5-TAP-IN) and ASd2 (cdc5-N209A-TAP-IN) (Table S1, Supplementary material).
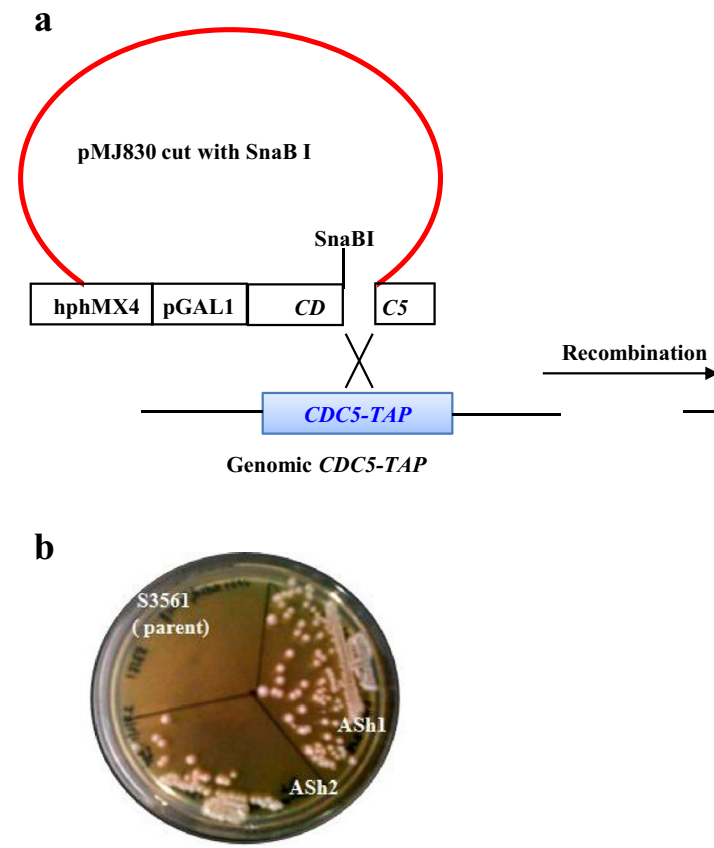

Fig. 2 a Schematic representation of the strategy used for construction of CDC5-TAP-IN strain by integration of SnaB I digested pMJ830 plasmid containing $p G A L l-C D C 5$ in the yeast cells (details in the text). b Growth of transformants ASh1 ( $p G A L 1-C D C 5-T A P)$ and ASh2 ( $p G A L 1-c d c 5-N 209 A-T A P)$ on hygromycin selection medium. The untransformed S3561 parent showed no growth 
a

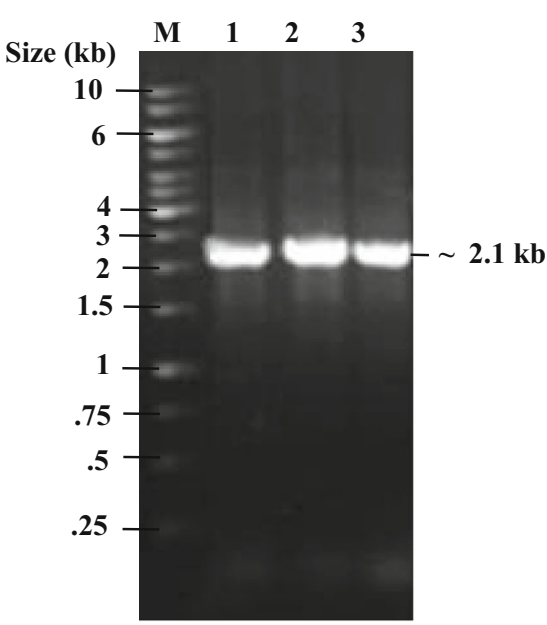

b

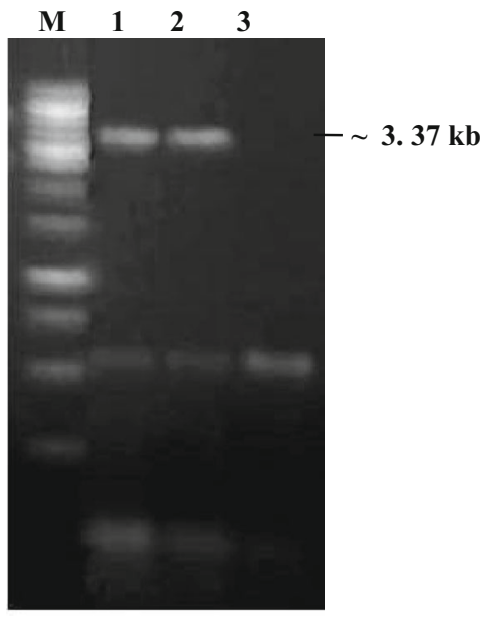

c

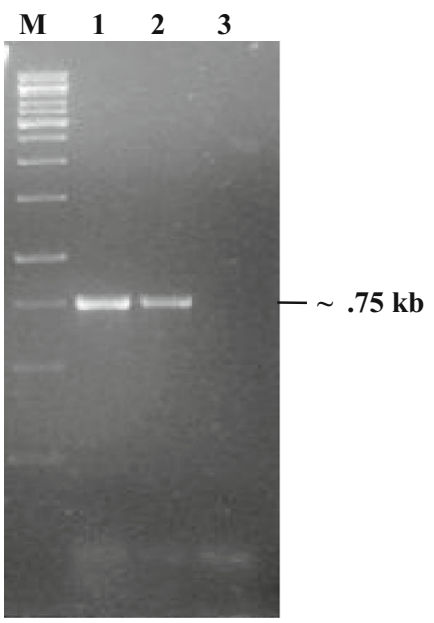

Fig. 3 Confirmation of Cdc5-TAP-IN and $c d c 5-N 209 A-I N$ transformants by PCR. Forward primers as $111 \mathrm{f}$, as $65 \mathrm{f}$, and reverse primers as106r, 113r, respectively, were used for the verification of CDC5-IN and $c d c 5-N 209 A-I N$ transformants by PCR. a PCR verification with primer set as111f - as113r. $M$ size marker (1 kb ladder); lanes 1, 2, 3 S3561 (wild-type), ASh1 and ASh2 (transformants), respectively, show $(\sim 2.1 \mathrm{~kb})$ PCR products as expected for wild type and

\section{Inducible expression of Cdc5-TAP and cdc5-N209A- TAP}

The diploids CDC5-TAP-IN and $c d c 5-N 209 A-T A P-I N$ were incubated in sporulation medium to induce meiosis for $7 \mathrm{~h}$, followed by the addition of inducer (ED). Inducible expression of CDC5-TAP and cdc5-N209A-TAP was confirmed by Western analysis using anti-TAP tag antibodies (Fig. 4). On the other hand, the expression of Cdc5TAP or cdc5-N209A-TAP was absent in the uninduced samples (-ED) (Fig. 4).

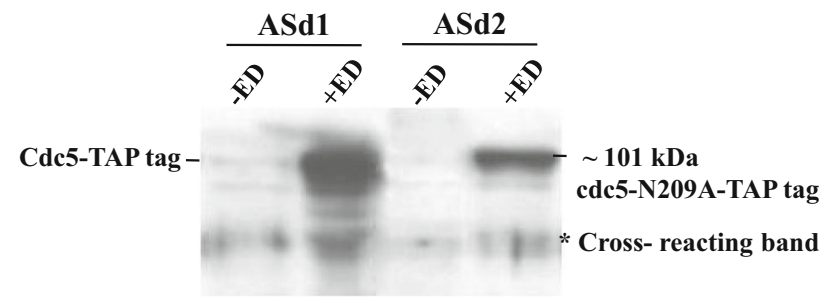

Fig. 4 Verification of inducible expression of Cdc5-TAP and cdc5N209A-TAP kinase-dead proteins in meiotic cultures. Western analysis of the meiotic protein extracts of ASd1 and ASd2 in the absence $(-\mathrm{ED})$ and presence $(+\mathrm{ED})$ of inducer with anti-TAP antibodies. Samples were collected after $2 \mathrm{~h}$ of induction. A specific band corresponding to the size of Cdc5-TAP or cdc5-N209A-TAP fusion proteins $(\sim 101 \mathrm{kDa})$ is observed upon ED addition transformants. b, $\mathbf{c}$ PCR Confirmation of transformants with primer sets as65f-as $106 \mathrm{r}$ and as65f-as $113 \mathrm{r}$, respectively. $M$ size marker (1 kb ladder), Lanes 1, 2 ASh1 and ASh2, respectively; lane 3 S3561 (wild-type). In b, c both transformants ASh1 and ASh2 show PCR products of expected size of $\sim 3.37$ and $\sim 0.75 \mathrm{~kb}$, respectively, whereas, no PCR product was obtained for the untransformed wildtype S3561

\section{Conclusion}

In our study, we have successfully generated $S$. cerevisiae strains that were engineered within an inducible Cdc5-TAP construct for the purification of substrates binding $\mathrm{Cdc} 5$ kinase. The inducible expression of CDC5-TAP and its kinase-dead version system was validated by western analysis. Thus, in our study we have constructed $n d t 804$ CDC5-IN (pGAL1-CDC5-TAP) strain to induce and express target protein during pachytene of meiosis-I.

The TAP-tagging CDC5 would provide a scope to aim the targets that are phosphorylated during pachytene exit. Thus, in future, the Cdc5-IN system will be used to pulldown the substrates of $\mathrm{Cdc} 5$ during meiosis. The approach can be extended to study other cellular processes in yeast meiosis. This approach shall provide opportunity to study organizational, functional, and interaction patterns of proteome involved during pachytene exit. In future, the combination of estradiol-inducible expression and TAP-tag systems could be used to analyze the proteome during specific stages of meiosis.

Acknowledgments We thank Dr. Wolfgang Zachariae, Max Planck Institute of Molecular Cell Biology and Genetics, Germany for providing genomic-tagged CDC5-TAP yeast strains. We acknowledge the research funding to Dr. Sourirajan and research fellowship to Ms. Vaid provided by the Department of Biotechnology (DBT), Government of India under the project BT/Bio-CARe/01/616. The authors would like to acknowledge Shoolini University for infrastructural support in the research work. 


\section{Compliance with ethical standards}

Conflict of interest The authors hereby declare that they have no conflict of interest.

Open Access This article is distributed under the terms of the Creative Commons Attribution 4.0 International License (http:// creativecommons.org/licenses/by/4.0/), which permits unrestricted use, distribution, and reproduction in any medium, provided you give appropriate credit to the original author(s) and the source, provide a link to the Creative Commons license, and indicate if changes were made.

\section{References}

Allers T, Lichten M (2001) Differential timing and control of noncrossover and crossover recombination during meiosis. Cell 106:47-57

Attner MA, Miller MP, Ee LS et al (2013) Polo kinase CDC5 is a central regulator of the meiosis I. Proc Natl Acad Sci USA 110:14278-14283

Benjamin KR, Zhang C, Shokat KM et al (2003) Control of landmark events in meiosis by the CDK Cdc28 and the meiosis-specific kinase Ime2. Genes Dev 17:1524-1539

Börner GV, Cha RS (2015) Analysis of meiotic recombination and homolog interaction during yeast meiosis. Cold Spring Harb Protoc 10:914-924

Bradford MM (1976) Rapid and sensitive method for the quantitation of microgram quantities of protein utilizing the principle of protein-dye binding. Anal Biochem 72:248-254

Chu S, Herskowitz I (1998) Gametogenesis in yeast is regulated by a transcriptional cascade dependent on Ndt80. Mol Cell 1:685-696

Clyne RK, Katis VL, Jessop L et al (2003) Polo-like kinase Cdc5 promotes chiasmata formation and cosegregation of sister centromeres at meiosis I. Nat Cell Biol 5:480-485

Dougherty WG, Cary SM, Parks TD (1989) Molecular genetic analysis of a plant virus polyprotein cleavage site: a model. Virology 171:356-364

Goyon C, Lichten M (1993) Timing of molecular events in meiosis in Saccharomyces cerevisiae: heteroduplex DNA is formed late in meiotic prophase. Mol Cell Biol 13:373-382

Hochwagen A, Amon A (2006) Checking your breaks: surveillance mechanisms of meiotic recombination. Curr Biol 16:R217-R228

Kane SM, Roth R (1974) Carbohydrate metabolism during ascospore development in yeast. J Bacteriol 118:8-14

Kapust RB, Tözsér J, Fox JD et al (2001) Tobacco etch virus protease: mechanism of autolysis and rational design of stable mutants with wild-type catalytic proficiency. Protein Eng 14:993-1000
Lee BH, Amon A (2003a) Polo kinase-meiotic cell cycle coordinator. Cell Cycle 2:400-402

Lee BH, Amon A (2003b) Role of Polo-like kinase CDC5 in programming meiosis I chromosome segregation. Science 300:482-486

Lee KS, Park JE, Asano S, Park CJ (2005) Yeast polo-like kinases: functionally conserved multitask mitotic regulators. Oncogene 24:217-229

Lee KS, Park JE, Kang YH et al (2008) Mechanisms of mammalian polo-like kinase1 (Plk1) localization: self- versus non-selfpriming. Cell Cycle 7:141-145

Matos J, Lipp JJ, Bogdanova A et al (2008) Dbf4-dependent Cdc7 kinase links DNA replication to the segregation of homologous chromosomes in meiosis I. Cell 135:662-678

Nigg EA (1998) Polo-like kinases: positive regulators of cell division from start to finish. Curr Opin Cell Biol 10:776-783

Puig O, Caspary F, Rigaut G et al (2001) The tandem affinity purification (TAP) method: a general procedure of protein complex purification. Methods 24:218-229

Rigaut G, Shevchenko A, Rutz B et al (1999) A generic protein purification method for protein complex characterization and proteome exploration. Nat Biotechnol 17:1030-1032

Sambrook J, Fritsch EF, Maniatis T (2009) Molecular cloning: a laboratory manual. Cold Spring Harbor Laboratory Press, New York

Sharon G, Simchen G (1990) Mixed segregation of chromosomes during single-division meiosis of Saccharomyces cerevisiae. Genetics 125:475-485

Simchen G (2009) Commitment to meiosis: what determines the mode of division in budding yeast? BioEssays 31:169-177

Sourirajan A, Lichten M (2008) Polo-like kinase Cdc5 drives exit from pachytene during budding yeast meiosis. Genes Dev 22:2627-2632

Uhlen MB, Nilsson B, Guss M (1983) Gene fusion vectors based on the gene for Staphylococcal protein A. Gene 23:369-378

Vaid R, Sharma N, Chauhan S et al (2016) Functions of polo-like kinases: a journey from yeast to humans. Protein Pept Lett 23:185-197

Van Driessche BL, Tafforeau P, Hentges AM et al (2005) Additional vectors for PCR-based gene tagging in Saccharomyces cerevisiae and Schizosaccharomyces pombe using nourseothricin resistance. Yeast 22:1061-1068

Xu L, Ajimura M, Padmore R et al (1995) NDT80, a meiosis-specific gene required for exit from pachytene in Saccharomyces cerevisiae. Mol Cell Biol 15:6572-6581

Xu X, Song Y, Li Y et al (2010) The tandem affinity purification method: an efficient system for protein complex purification and protein interaction identification. Protein Expr Purif 72:149-156 